\title{
Alterações em variáveis limnológicas de manancial de Juiz de Fora devido ao uso da terra
}

\author{
Cézar H. B. Rocha ${ }^{1}$, Fabiano A. Freitas ${ }^{2} \&$ Thiago M. da Silva ${ }^{3}$ \\ ${ }^{1}$ Faculdade de Engenharia/ NAGEA/PGECOL/UFJF. Juiz de Fora, MG. Fone: (32) 2102-3414. E-mail: barra.rocha@gmail.com (Autor correspondente) \\ ${ }^{2}$ PGECOL/NAGEA/UFJF. Juiz de Fora, MG. E-mail: fabianoamarante29@gmail.com \\ ${ }^{3}$ PGECOL/NAGEA/UFJF. Juiz de Fora, MG. E-mail: martins.nagea@gmail.com
}

Palavras-chave:

recursos hídricos

bacia hidrográfica

sazonalidade

análise de componentes principais

\begin{abstract}
R E S U M O
Os processos que promovem alterações em variáveis limnológicas são amplos e necessitam de estudos que considerem as peculiaridades das bacias de contribuição, bem como o uso e cobertura de suas áreas. O presente artigo teve por objetivo comparar a dinâmica de parâmetros limnológicos da Represa Dr. João Penido - Juiz de Fora/MG em diferentes regimes pluviométricos, além de compreender os mecanismos atuantes na variação dos mesmos. O teste $t$ de Student verificou a ocorrência de variação sazonal significativa para os parâmetros: cor, turbidez, ferro, condutividade e demanda bioquímica de oxigênio. Através da Análise Fatorial/Análise de Componentes Principais foi excluída a variável $\mathrm{pH}$ por não contribuir para a variância total dos dados além de destacar, dentre as variáveis estudadas, as que mais influenciaram na qualidade da água. Foram utilizadas as quatro primeiras componentes que explicaram $71 \%$ da variância total dos dados indicando o escoamento superficial como determinante das variáveis da primeira e da segunda componentes; a contribuição orgânica não associada à precipitação, como sugestiva das variáveis da terceira componente e a dinâmica funcional intrínseca do ambiente aquático como indicativa da quarta componente.
\end{abstract}

Key words:

water resources

hydrographic basin

seasonality

principal component analysis

\section{Changes in limnological variables of water reservoir in Juiz de Fora due to land use}

\begin{abstract}
A B S T R A C T
The processes that promote changes in limnological variables are large and require studies that consider the peculiarities of the basins of contribution, as well as the use and coverage of their areas. This article aimed to compare the dynamics of limnological parameters of Dr. Joao Penido reservoir in Juiz de Fora - State of Minas Gerais in different rainfall regimes, as well as understanding the active mechanisms in the variation of the same ones. Student's t-test has revealed the occurrence of significant seasonal variation for the parameters: color, turbidity, iron, electrical conductivity and biochemical oxygen demand. Through factor analysis/principal components analysis variable $\mathrm{pH}$ was excluded for not contributing to the variance of all data, highlighting among the studied variables, which contributed more to influence water quality. The first four components were used which explained $71 \%$ of the total variance of the data, indicating the runoff as a determinant of the variables of the first and second components; the organic contribution, not associated with the precipitation, as suggestive of the variables of the third component; and intrinsic functional dynamics of the aquatic environment as indicative of the fourth component.
\end{abstract}

\section{INTRODUÇÃO}

Dentre os múltiplos usos dos recursos hídricos a utilização para fins de consumo humano e dessedentação de animais é prioritária, como assegurada por Lei Federal. Para isto é necessário garantir a qualidade assegurando sua adequação a esses fins. As variáveis limnológicas que inferem na qualidade da água sofrem grandes variações espaciais e temporais ressaltando-se a carência de um monitoramento sistemático (Andrade et al., 2007; Carmo et al., 2008). Tais alterações podem ser provenientes de processos naturais (sazonalidade, intempéries, dentre outros) ou antropogênicos (uso inapropriado da terra, descarga de efluentes, uso indiscriminado de defensivos agrícolas, são alguns exemplos).

Os corpos hídricos são sistemas complexos caracterizados como escoadouros naturais das áreas de drenagem que formam a bacia hidrográfica (Toledo \& Nicolella, 2002), justificando a assertiva de que a gestão dos recursos hídricos deve levar em consideração toda a bacia em questão. Segundo Fritzsons et al. (2003), a precipitação influencia na vazão com reflexos na qualidade da água, embora os autores destaquem a dificuldade de se estabelecer uma relação direta tendo em vista a complexidade dos processos envolvidos. Com base nessas perspectivas, o monitoramento dos parâmetros físicos, químicos e biológicos da água é necessário para uma gestão 
adequada deste recurso visando assegurar sua disponibilidade e qualidade.

Silva et al. (2009) afirmam que a mitigação dos impactos e a preservação da qualidade e dos usos múltiplos de um reservatório dependem de manejo integrado que, por sua vez, se ressente de um conhecimento aprofundado desses sistemas, incluindo informações sobre a qualidade de suas águas e do uso do solo em seu entorno.

O município de Juiz de Fora - MG, local em que se situa a Represa Dr. João Penido, objeto deste estudo, apresenta uma sazonalidade bem definida. Segundo a Lei Municipal $\mathrm{n}^{\circ}$ 9.811/2000, Plano Diretor de Desenvolvimento Urbano PDDU (Juiz de Fora, 2000) a estação chuvosa vai de outubro a abril com temperaturas mais elevadas e maiores precipitações pluviométricas e a estação seca se prolonga de maio a setembro, período mais frio e com menor presença de chuvas.

A bacia de contribuição do manancial, embora considerada área urbana do município de Juiz de Fora, apresenta baixo adensamento demográfico e características eminentemente rurais, como a criação de bovinos, suínos e atividades hortigranjeiras, dentre outras (Juiz de Fora, 2000).

A Lei Municipal n 6.910/1986 (Juiz de Fora, 1986) institui a Bacia Hidrográfica da Represa Dr. João Penido como Zona Especial, sujeita a regime urbanístico específico que visa à preservação dos seus recursos naturais e à proteção ambiental e ecológica. Outra idiossincrasia jurídica que envolve esta bacia é a delimitação de suas áreas de preservação que seguem determinações preconizadas no artigo $3^{\circ}$ da Lei Municipal $n^{\circ}$ 6087/1981 (Juiz de Fora, 1981), com destaque para os incisos II e III que preveem, respectivamente, a faixa de proteção de $100 \mathrm{~m}$ de largura em projeção horizontal, a partir da cota 744 $\mathrm{m}$ (nível máximo da represa) e $50 \mathrm{~m}$ a partir do leito menor, em cada uma das margens dos cursos d'água.

O grande número de variáveis limnológicas passíveis de serem monitoradas resulta em uma matriz de ampla dimensão com difícil interpretação. As abordagens multivariadas como a análise fatorial $(\mathrm{AF})$ e a análise de componentes principais (ACP) são úteis para extrair informações significativas promovendo a redução do número de variáveis com o mínimo de perdas das informações (Andrade et al., 2007; Lial et al., 2008; Bernardi et al., 2009; Mendonça \& Souza, 2011; Guedes et al., 2012).

A Represa Dr. João Penido é o principal manancial da cidade ficando, eventualmente, sobrecarregada em épocas de estiagem, carecendo de investimentos no sentido de explorar, de forma mais eficiente, o potencial de sua bacia hidrográfica (Ribeiro \& Pizzo, 2011). Ressalta-se, portanto, que o manancial possui corpo bibliográfico escasso disponível que possa contribuir com a gestão de suas águas. Buscando diminuir esta lacuna objetivou-se compreender a dinâmica das variáveis limnológicas nos diferentes regimes pluviométricos e identificar os processos mais atuantes na sua variação.

\section{Material e Métodos}

As informações limnológicas utilizadas foram cedidas pela Companhia de Saneamento Municipal - CESAMA e se referem às análises da água na captação da Represa Dr. João Penido com coordenadas geográficas: $21^{\circ} 41^{\prime} 03,3^{\prime \prime} \mathrm{S}$ e $43^{\circ} 24^{\prime} 12,68^{\prime \prime}$ W (Datum WGS-84). A CESAMA afirma que, atualmente, este manancial supre a necessidade de $50 \%$ da cidade de Juiz de Fora podendo chegar a até $65 \%$; sua construção se deu com o barramento do ribeirão dos Burros em 1934 contando também com os córregos Grama e Vista Alegre. A área total de drenagem da bacia de contribuição é de aproximadamente $68 \mathrm{~km}^{2}$. Utilizou-se o software ESRI ${ }^{\circledast}$ ArcMap $^{\text {TM }} 9.3$ para a elaboração do Mapa de Localização, conforme a Figura 1.
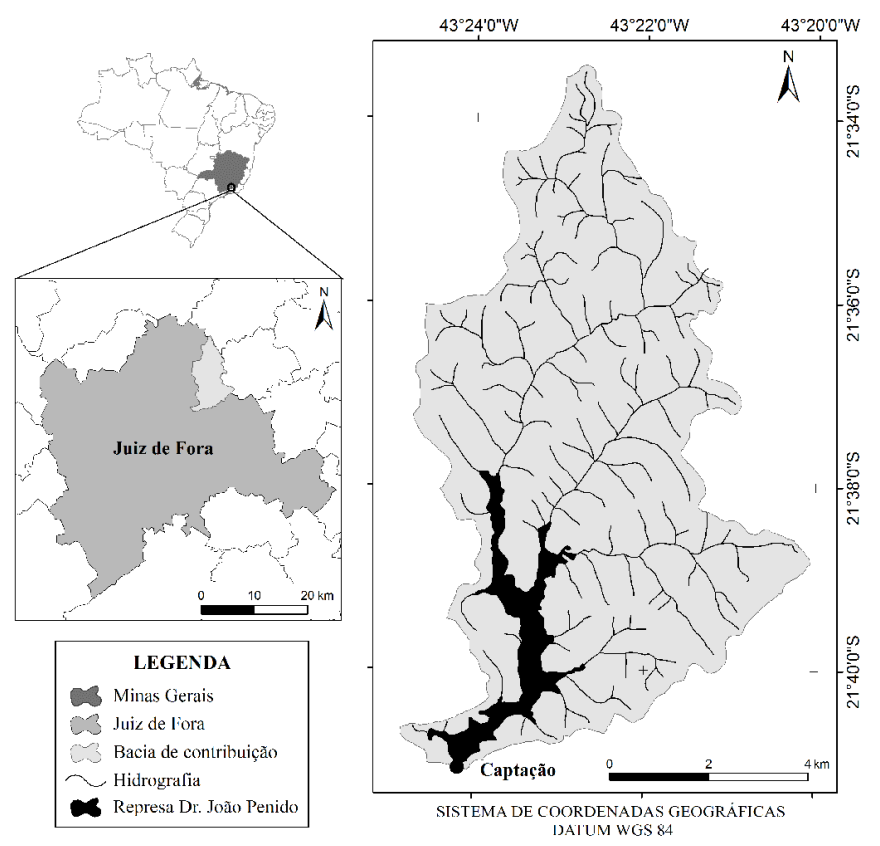

Fonte: Instituto Brasileiro de Geografia e Estatística - IBGE

Figura 1. Mapa de localização da área de estudo

Machado (2006) considera a Lei Municipal n 6.087/1981 (Juiz de Fora, 1981) um avanço na proteção da represa, mas apresenta, como ressalva, que ela é baseada em critérios pouco científicos estando mais ligada ao processo de ocupação do que à manutenção das qualidades mínimas de suas águas. Tal ponderação ficou ainda mais evidente com a alteração do seu artigo $9^{\circ}$ pela Lei Municipal no 11.817/2009 (Juiz de Fora, 2009) que possibilita a "implantação de infraestrutura de alto interesse público" em áreas consideradas de preservação, um retrocesso.

Foram analisados os seguintes parâmetros: $\mathrm{pH}$, cor, turbidez, ferro (Fe), fósforo total (PT), alcalinidade $\mathrm{HCO}_{3}^{-}$, cloreto, dureza, oxigênio consumido (OC), oxigênio dissolvido (OD), condutividade, demanda bioquímica de oxigênio $\left(\mathrm{DBO}_{5,20}\right)$ e nitrogênio total (NT) referente às medições mensais realizadas entre o período de 2006 a 2012.

Os dados foram divididos em duas estações: estação seca e chuvosa, como definido pela Lei Municipal no 9.811/2000 (Juiz de Fora, 2000). Procedeu-se anualmente ao cálculo das médias de cada parâmetro para as duas estações e se verificou a ocorrência de normalidade (Teste Kolmogorov-Smirnov). Foi aplicado o teste $t$ de Student, com o auxílio do Microsoft Office Excel 2007 para verificar a ocorrência de variação estatisticamente significativa $(\mathrm{p}<0,05)$ entre as médias 
históricas das matrizes formadas com os dados de seca e chuva dos sete anos monitorados (2006 a 2012). Alguns dados não foram mensurados durante vários meses de um mesmo ano, impossibilitando calcular uma média referente à estação daquele ano motivo pelo qual para os parâmetros NT e PT, foram excluídos da matriz os dados de 2011; para a dureza, os dados de 2012; para o OC, 2006 e por fim, para o parâmetro OD foram eliminados os dados de 2006, 2007 e 2011.

Verificou-se a adequação dos dados à AF por meio dos testes de Kaiser-Meyer-Olkin (KMO) e esfericidade de Bartlett. As análises multivariadas (AF/ACP) foram realizadas no software SPSS $^{\circledast} 15.0$ e consistiram em três etapas descritas por Toledo \& Nicolella (2002): elaboração da matriz de correlação; extração dos fatores comuns com possível redução das variáveis explicativas e rotação dos eixos relativos aos fatores comuns visando facilitar e simplificar a interpretação.

Os dados originais, em forma de matriz, foram expressos por $\mathrm{X}=(\mathrm{Xi}, \mathrm{j})$ em que $\mathrm{i}=1$...n amostragens $(25)$ e $\mathrm{j}=1$...p variáveis limnológicas (12). As amostragens abrangeram os anos de 2006 a 2012 ressaltando-se, entretanto, ausência de dados em vários meses, os quais foram desconsiderados nas análises.

O coeficiente de correlação superior a 0,5 , como sugerido por Cohen (1988) e Helena et al. (2000) expressa uma forte correlação entre as variáveis limnológicas e valores entre 0,3 e 0,49, expressam uma correlação moderada (Cohen, 1988). Para extração das componentes principais utilizou-se a matriz de correlação visando eliminar o problema de escalas e unidades diferenciadas em que as variáveis foram medidas. A principal característica da ACP, além da ortogonalidade, é que as componentes principais são obtidas em ordem decrescente de máxima variância. Assim, a primeira componente explica o máximo da variabilidade total dos dados; a segunda, explica o máximo de variabilidade dos dados restantes, não correlacionados com a primeira e assim sucessivamente (Andrade et al., 2007; Guedes et al., 2012).

Os resultados da ACP nem sempre são de fácil interpretação. Para suplantar tal limitação adota-se procedimento de rotação ortogonal da matriz das cargas fatoriais que possibilita melhor interpretação dos fatores ao redistribuir a variância explicada pelas componentes, não alterando na variância acumulada do conjunto de componentes. A utilização da rotação ortogonal pelo método Varimax, objetiva um ajuste melhor ao modelo fatorial possível de explicação, sendo frequentemente utilizada em estudos de qualidade de água e processos hidrológicos (Aprile \& Farias, 2001; Andrade et al., 2007; Girão et al., 2007; Guedes et al., 2012). Maiores detalhes sobre o assunto podem ser encontrados em Hair et al. (2007).

\section{Resultados e Discussão}

Os resultados das análises preliminares que visaram testar a adequacidade dos dados à AF/ACP, possibilitaram o emprego de tal metodologia. Ao testar a hipótese de que a matriz de correlação é uma matriz identidade, teste de esfericidade de Bartlett $(\mathrm{p}=0,001)$ foi descartada a hipótese nula verificando-se a existência de correlações significativas entre as variáveis. Outro teste utilizado foi o índice de adequação da amostra (KMO) que, segundo Hair et al. (2007), quantifica o grau de intercorrelação entre as variáveis. $\mathrm{O}$ resultado encontrado foi $\mathrm{KMO}=0,611$, o que permite a aplicação da análise.

Informações relevantes sobre a dinâmica das variáveis podem ser obtidas através da matriz de correlação. Os parâmetros limnológicos que possuíram maior número de correlações (Tabela 1) tiveram maior importância na composição das componentes principais (CPs). No processamento da AF/ ACP foram extraídas, a partir da matriz de correlação, as comunalidades que representam o nível de associação entre a variável e os fatores extraídos, ou seja, a porcentagem da variância da variável explicada pelos fatores utilizados (Toledo \& Nicolella, 2002). Valores abaixo de 0,5 indicam que a variável deve ser eliminada e a AF/ACP refeita (Figueiredo Filho \& Silva Júnior, 2010). Norteado por tais considerações, eliminou-se o $\mathrm{pH}$ da análise.

As quatro primeiras componentes explicaram $71 \%$ da variância total dos dados. Embora não exista um critério consensual sobre o número de fatores que devem ser extraídos na análise, o critério de Kaiser sugere a extração dos fatores com valor de eigenvalue (autovalor) acima de um (Figueiredo Filho \& Silva Júnior, 2010). Já Hair et al. (2007) sugerem que o número de componentes que represente um percentual de $60 \%$ da variância acumulada reflete uma ideia aceitável da variância original.

Tabela 1. Matriz de correlação das variáveis limnológicas da Represa Dr. João Penido

\begin{tabular}{|c|c|c|c|c|c|c|c|c|c|c|c|c|}
\hline & Cor & Turb. & $\mathrm{Fe}$ & PT & Alcalin. & Cloreto & Dureza & OC & OD & Condut. & $\mathrm{DBO}_{5,20}$ & NT \\
\hline Cor & 1,00 & & & & & & & & & & & \\
\hline Turb. & 0,44 & 1,00 & & & & & & & & & & \\
\hline $\mathrm{Fe}$ & 0,77 & 0,35 & 1,00 & & & & & & & & & \\
\hline PT & $-0,37$ & $-0,15$ & $-0,25$ & 1,00 & & & & & & & & \\
\hline Alcalin. & 0,44 & 0,30 & 0,30 & $-0,07$ & 1,00 & & & & & & & \\
\hline Cloreto & 0,09 & 0,04 & 0,06 & 0,04 & 0,36 & 1,00 & & & & & & \\
\hline Dureza & 0,22 & 0,11 & 0,18 & $-0,20$ & 0,37 & 0,35 & 1,00 & & & & & \\
\hline OC & 0,07 & 0,31 & 0,08 & $-0,36$ & 0,08 & 0,25 & 0,05 & 1,00 & & & & \\
\hline OD & 0,57 & 0,36 & 0,46 & $-0,25$ & 0,32 & 0,23 & $-0,03$ & 0,48 & 1,00 & & & \\
\hline Condut. & 0,62 & 0,46 & 0,51 & $-0,13$ & 0,39 & 0,20 & 0,14 & 0,12 & 0,35 & 1,00 & & \\
\hline $\mathrm{DBO}_{5,20}$ & 0,18 & 0,36 & 0,25 & 0,19 & 0,37 & 0,05 & 0,24 & $-0,10$ & 0,05 & 0,46 & 1,00 & \\
\hline NT & 0,37 & $-0,22$ & 0,36 & $-0,21$ & 0,17 & 0,44 & 0,46 & 0,01 & 0,10 & 0,22 & 0,07 & 1,00 \\
\hline
\end{tabular}

Legenda: Cor (uH); Turb. - Turbidez (uT); Fe - Ferro ( $\mathrm{mg} \mathrm{L}^{-1}$ ); PT - Fósforo Total (mg L-1); Alcalin. - Alcalinidade $\mathrm{HCO}_{3}^{-}\left(\mathrm{mg} \mathrm{L}^{-1}\right)$; Cloreto (mg L-1); Dureza (mg L-1); OC - Oxigênio Consumido

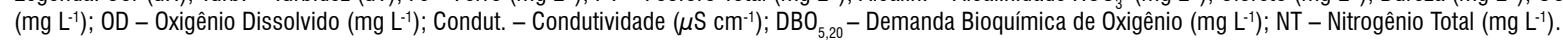


A Tabela 2 apresenta a matriz não rotacionada de pesos fatoriais que indicam a contribuição que cada variável possui na componente principal. Observa-se alta concentração de explicação da variância total dos dados na primeira componente (CP1) e variáveis com elevado peso fatorial em mais de uma CP. Comportamento semelhante foi verificado por outros autores (Helena et al., 2000; Andrade et al., 2007; Guedes et al., 2012). Para suplantar esta dificuldade, procedeu-se à rotação Varimax.

Tabela 2. Matriz de pesos fatoriais das variáveis liminológicas nas quatro componentes principais (CP) selecionadas

\begin{tabular}{lrrrr}
\hline \multicolumn{1}{c}{ Variável } & CP 1 & CP 2 & CP 3 & CP 4 \\
Cor & 0,84 & $-0,12$ & 0,01 & $-0,38$ \\
Turbidez & 0,58 & $-0,45$ & 0,29 & 0,27 \\
Fe & 0,75 & $-0,08$ & 0,05 & $-0,42$ \\
P Total & $-0,37$ & 0,21 & 0,58 & 0,27 \\
Alcalinidade & 0,63 & 0,22 & 0,20 & 0,28 \\
Cloreto & 0,38 & 0,50 & $-0,28$ & 0,53 \\
Dureza & 0,42 & 0,61 & $-0,11$ & 0,09 \\
OC & 0,33 & $-0,37$ & $-0,53$ & 0,51 \\
OD & 0,65 & $-0,40$ & $-0,24$ & 0,14 \\
Condutividade & 0,74 & $-0,06$ & 0,31 & $-0,05$ \\
DBO & 0,41 & 0,17 & 0,69 & 0,16 \\
N Total & 0,43 & 0,65 & $-0,34$ & $-0,28$ \\
Autovalor & 3,94 & 1,73 & 1,61 & 1,25 \\
\% variância explicada & 32,91 & 14,41 & 13,46 & 10,46 \\
\% variância acumulada & 32,91 & 47,32 & 60,79 & 71,26 \\
\hline CP - Componente Principal & & & &
\end{tabular}

A primeira componente após a aplicação do algoritmo Varimax (FV1) explicou $21,79 \%$ da variância total dos dados e teve como variáveis mais expressivas, a cor e o Fe, além do PT, com menor significância nesta componente, mantendo-se próximo do valor adotado $(0,5)$ para validar sua participação de destaque na FV1 (Tabela 3). Dentre as variáveis estudadas a cor e o Fe foram as que apresentaram maior número de correlações possuindo entre si, inclusive, o maior coeficiente de Pearson da matriz (Tabela 1). Isto se justifica pelo fato do Fe se apresentar na forma dissolvida na água (Sperling, 2005). Os resultados obtidos pelo teste $t$ de Student destacam variação sazonal para a cor e o Fe com elevação de suas médias na estação chuvosa (Tabela 4).

Tabela 3. Matriz de pesos fatoriais das quatro componentes principais após rotação Varimax

\begin{tabular}{lrrrr}
\hline Voriável & FV 1 & FV 2 & FV 3 & FV 4 \\
Cor & 0,88 & 0,26 & 0,13 & 0,13 \\
P Total & 0,83 & 0,23 & 0,11 & 0,04 \\
DBO & $-0,54$ & 0,39 & $-0,09$ & $-0,38$ \\
Turbidez & 0,08 & 0,81 & 0,11 & $-0,19$ \\
Alcalinidade & 0,27 & 0,61 & $-0,20$ & 0,46 \\
Cloreto & 0,19 & 0,56 & 0,42 & 0,18 \\
NT & $-0,15$ & 0,15 & 0,76 & 0,34 \\
Dureza & 0,41 & $-0,18$ & 0,76 & $-0,16$ \\
OC & 0,12 & 0,14 & 0,73 & $-0,04$ \\
OD & 0,01 & $-0,07$ & 0,09 & 0,89 \\
Condutividade & 0,46 & 0,16 & 0,01 & 0,65 \\
Autovalor & 0,54 & 0,57 & 0,11 & 0,12 \\
\% variância explicada & 2,61 & 2,08 & 2,02 & 1,83 \\
\% variância acumulada & 21,79 & 17,35 & 16,82 & 15,28 \\
\hline FV - Fator Varimax & 21,79 & 39,14 & 55,97 & 71,26 \\
\hline
\end{tabular}

Tabela 4. Resultados do teste t Student e valores de média e desvio padrão das variáveis monitoradas

\begin{tabular}{|c|c|c|c|}
\hline \multirow[t]{2}{*}{ Variável } & \multirow{2}{*}{$\begin{array}{l}\text { Teste t Student } \\
\text { (valor de p) }\end{array}$} & \multicolumn{2}{|c|}{$\begin{array}{c}\text { Média e desvio } \\
\text { padrão (2006-2012) }\end{array}$} \\
\hline & & Seca & Chuva \\
\hline Cor (uH) & 0,0002 & $30,21 \pm 8,49$ & $56,49 \pm 12,34$ \\
\hline Turbidez (uT) & 0,0004 & $4,40 \pm 0,85$ & $6,11 \pm 1,24$ \\
\hline $\mathrm{Fe}\left(\mathrm{mg} \mathrm{L}^{-1}\right)$ & 0,0004 & $0,61 \pm 0,24$ & $1,41 \pm 0,22$ \\
\hline P Total (mg L-1) & 0,940 & $0,35 \pm 0,18$ & $0,35 \pm 0,11$ \\
\hline Alcalinidade $\left(\mathrm{mg} \mathrm{L}^{-1}\right)$ & 0,327 & $10,77 \pm 4,22$ & $9,75 \pm 1,79$ \\
\hline Cloreto (mg L-1) & 0,356 & $5,37 \pm 3,08$ & $4,93 \pm 2,35$ \\
\hline Dureza (mg L $\left.{ }^{-1}\right)$ & 0,304 & $8,31 \pm 1,55$ & $8,97 \pm 1,83$ \\
\hline $\mathrm{OC}\left(\mathrm{mg} \mathrm{L}^{-1}\right)$ & 0,198 & $2,49 \pm 0,49$ & $3,04 \pm 0,77$ \\
\hline $\mathrm{OD}\left(\mathrm{mg} \mathrm{L}^{-1}\right)$ & 0,608 & $5,48 \pm 1,38$ & $5,94 \pm 0,99$ \\
\hline Condutividade $\left(\mu \mathrm{S} \mathrm{cm}^{-1}\right)$ & 0,001 & $18,44 \pm 1,55$ & $21,32 \pm 0,82$ \\
\hline $\mathrm{DBO}_{5,20}\left(\mathrm{mg} \mathrm{L}^{-1}\right)$ & 0,010 & $7,22 \pm 2,69$ & $18,71 \pm 4,77$ \\
\hline N Total $\left(\mathrm{mg} \mathrm{L}^{-1}\right)$ & 0,312 & $1,69 \pm 1,20$ & $1,15 \pm 0,36$ \\
\hline
\end{tabular}

Os parâmetros determinantes da segunda componente (FV2) que explicaram $17,35 \%$ da variância total dos dados, foram a $\mathrm{DBO}_{5,20}$, turbidez e a alcalinidade. O comportamento da variável alcalinidade assemelhou-se ao do fósforo na FV1, inclusive por não apresentar variação sazonal. Sperling (2005) afirma que a alcalinidade da água pode originar-se da reação com o $\mathrm{CO}_{2}$ atmosférico ou proveniente da decomposição da matéria orgânica, o que corrobora com os valores encontrados de correlação moderada entre alcalinidade e as demais variáveis de destaque da FV2. A $\mathrm{DBO}_{5,20}$ e a turbidez estão relacionadas ao aporte de sólidos na água.

Na FV3 se destacam, com elevados coeficientes, os parâmetros cloreto, NT e dureza. O cloreto é proveniente da dissolução de sais sendo resultado do intemperismo de rochas ou de excrementos presentes nos esgotos (Sperling, 2005). A correlação moderada com o NT pode ser justificada pelo fato das variáveis terem origem antrópica comum. A carga de matéria orgânica associada ao esgoto doméstico é rica em nutrientes dentre eles diversos compostos nitrogenados e o próprio cloreto. A dureza da água se deve à presença de minerais, sobremaneira cálcio e magnésio que, normalmente, estão associados aos carbonatos, sulfatos e cloretos justificando a correlação moderada entre dureza e cloreto (Tabela 1). A correlação entre essas variáveis aponta para uma fonte comum, semelhante à observada por Andrade et al. (2007). É provável que esta fonte seja pontual, uma vez que os resultados do teste t de Student (Tabela 4) não indicaram diferenças significativas entre as médias sazonais.

Os maiores coeficientes de correlação das variáveis com a FV4 foram observados para o OD e OC, variáveis complexas que refletem toda a dinâmica dos ecossistemas aquáticos dentre elas a produtividade e o consumo. A FV4 explicou 15,28\% da variância total dos dados e as variáveis representantes desta componente não demonstraram ser influenciadas significativamente pela precipitação.

O comportamento das variáveis e sua distribuição pelas componentes permitem verificar as relações que apresentam entre si. Desta forma e como feito por outros autores (Helena et al., 2000; Andrade et al., 2007; Guedes et al., 2012), é possível atribuir um nome que represente as características comuns 
entre tais variáveis de cada componente; logo, a FV1 reflete a influência de sólidos dissolvidos associados à lixiviação pelo fato das variáveis com os maiores coeficientes terem apresentado diferenças significativas entre as estações de seca e chuva. A FV2 que se associa mais fortemente à presença de sólidos suspensos foi determinada pela turbidez e $\mathrm{DBO}_{5,20}$, variáveis com incremento de suas médias na estação chuvosa (Tabela 4). Os elevados coeficientes de cloreto e nitrogênio na FV3 caracterizam a influência do lançamento de esgotos na área da bacia. A FV4 representa fatores intrínsecos da dinâmica funcional do ambiente aquático em questão. $\mathrm{O}$ parâmetro condutividade apresentou correlações com variáveis tanto da FV1 quanto da FV2, além de variação sazonal, o que pode justificar sua presença em mais de uma componente.

Os resultados obtidos pelo teste $t$ de Student apontaram as variáveis com diferença sazonal significativa; contudo, não se descartou a hipótese de que outras variáveis também sofram interferência da precipitação uma vez que é difícil mensurar temporalmente as influências das chuvas sobre as variáveis limnológicas (Fritzsons et al., 2003). Acrescenta-se o fato das amostras coletadas serem provenientes da captação cuja qualidade da água pode ser resultado, dentre outros processos, do tamponamento ao longo do curso hídrico e da decantação na represa, semelhante ao descrito por Carvalho et al. (2000); Padial et al. (2009).

Tendo em vista que as componentes que mais explicaram a variância total dos dados (FV1 e FV2) são aquelas influenciadas pelo transporte e pela lixiviação de materiais, a redução da cobertura vegetal com exposição do solo e o manejo inadequado são os principais fatores de alteração da qualidade da água na captação deste manancial. Resultado semelhante foi encontrado por Vanzela et al. (2010) ao concluir que áreas habitadas, agricultadas e matas degradadas reduziram a qualidade das águas da bacia hidrográfica. Já Tundisi \& Tundisi (2010) são enfáticos ao afirmar que a remoção da vegetação aumenta o transporte de sólidos e a condutividade degradando o manancial e aumentando os custos de tratamento da água para abastecimento.

A ocupação das margens da Represa Dr. João Penido, com ausência de redes de coleta e tratamento de esgotos e em desconformidade com a legislação sobre Áreas de Preservação Permanente (APP) e a Lei Municipal 6.087/1981 (Juiz de Fora, 1981), também contribuíram para a degradação da qualidade das águas como observado pela FV3.

Carneiro et al. (2010) afirmam haver uma desarticulação entre os instrumentos de gerenciamento dos recursos hídricos e os de planejamento do uso do solo, marcada pelo forte grau de informalidade e até mesmo ilegalidade na ocupação do solo. Este fato é nitidamente observado na bacia hidrográfica do manancial objeto deste estudo.

\section{Conclusões}

1. A utilização do teste $t$ de Student confirmou a necessidade de estudos que contemplem diferentes estações (seca e chuva), tendo em vista a variação observada para importantes parâmetros limnológicos em virtude da pluviosidade.

2. A análise fatorial/análise de componentes principais promoveu a redução do número de variáveis de qualidade da água facilitando a interpretação dos dados que mais influenciam na dinâmica do manancial.

3. A análise permitiu a seleção de quatro componentes principais que explicaram $71 \%$ da variação total dos dados indicando o escoamento superficial como principal determinante das variáveis que compõem a primeira e a segunda componentes; a contribuição orgânica, não associada à precipitação como sugestiva das variáveis da terceira componente e a dinâmica funcional intrínseca do manancial refletindo na última componente.

\section{Agradecimentos}

Os autores agradecem à Companhia de Saneamento Municipal (CESAMA) pelos dados cedidos; ao Conselho Nacional de Desenvolvimento Científico e Tecnológico (CNPq) pelo financiamento da pesquisa; à Coordenação de Aperfeiçoamento de Pessoal de Nível Superior (CAPES) e à Universidade Federal de Juiz de Fora (UFJF) pelas bolsas concedidas.

\section{Literatura Citada}

Andrade, E. M. de; Araújo, L. de F. P.; Rosa, M. de F.; Disney, W.; Alves, A. B. Seleção dos indicadores da qualidade das águas superficiais pelo emprego da análise multivariada. Engenharia Agrícola, v.27, p.683-690, 2007.

Aprile, F. M.; Farias, V. P. Avaliação da qualidade da água da bacia do Rio Goiana, Pernambuco - Brasil. Bioikos, v.15, p.109-114, 2001.

Bernardi, J. V. E.; Lacerda, L. D.; Dórea, J. G.; Landim, P. M. B.; Gomes, J. P. O.; Almeida, R.; Manzatto, A. G.; Bastos, W. R. Aplicação da análise das componentes principais na ordenação dos parâmetros físico-químicos no alto Rio Madeira e afluentes, Amazônia Ocidental. Geochimica Brasiliensis, v.23, p.79-90, 2009.

Carmo, R. F.; Bevilacqua, P. D.; Bastos, R. K. X. Vigilância da qualidade da água para consumo humano: Abordagem qualitativa da identificação de perigos. Engenharia Sanitária e Ambiental, v.13, p.426-434, 2008.

Carneiro, P. R. F.; Cardoso, A. L.; Zampronio, G. B.; Martingil, M. de C. A gestão integrada de recursos hídricos e do uso do solo em bacias urbano-metropolitanas: O controle de inundações na bacia dos rios Iguaçu/Sarapuí, na Baixada Fluminense. Ambiente \& Sociedade, v.13, p.29-49, 2010.

Carvalho, A. R.; Schlittler, F. H. M.; Tornisielo, V. L. Relações da atividade agropecuária com parâmetros físicos químicos da água. Química. Nova, v.23, p.618-622, 2000.

Cohen, J. Statistical power analysis for the behavioral sciences. 2.ed. Hillsdale, New Jersey: Lawrence Erlbaum Associates, Publishers, 1988. 567p. 
Figueiredo Filho, D. B.; Silva Júnior, J. A. da. Visão além do alcance: Uma introdução à análise fatorial. Opinião Pública, v.16, p.160-185, 2010.

Frintzons, E.; Hind, E. C.; Mantovani, L. E.; Rizzi, N. E. As alterações da qualidade da água do Rio Capivari com o deflúvio: um instrumento de diagnóstico de qualidade ambiental. Engenharia Sanitária e Ambiental, v.8, p.239248, 2003.

Girão, E. G.; Andrade, E. M.; Rosa, M. de F. Araújo, L. de F. P.; Meireles, A. C. M. Seleção dos indicadores da qualidade de água no Rio Jaibaras pelo emprego da análise de componente principal. Revista Ciência Agronômica, v.38, p.17-24, 2007.

Guedes, H. A. S.; Silva, D. D.; Elesbon, A. A. A.; Ribeiro, C. B. M.; Matos, A. T.; Soares, J. H. P. Aplicação da análise estatística multivariada no estudo da qualidade da água do Rio Pomba, MG. Revista Brasileira de Engenharia Agrícola e Ambiental, v.16, p.558-563, 2012.

Hair, J. J. F; Anderson, R. E.; Tatham, R. L.; Black, W. C. Análise multivariada de dados. 5.ed. Porto Alegre: Bookman, 2007. $593 p$

Helena, B.; Pardo, R.; Vega, M.; Barrado, E.; Fernández, J. M.; Fernández, L. Temporal evolution of groundwater composition in an alluvial aquifer (Pisuerga River, Spain) by principal component analysis. Water Research, v.34, p.807-816, 2000.

Juiz de Fora. Lei nº 6.087 de 04 de dezembro de 1981. Dispõe sobre o parcelamento, uso e ocupação do solo, na área da bacia hidrográfica da Represa Dr. João Penido, em Juiz de Fora e dispõe outras providências. <http://www. leismunicipais.com.br/legislacao-de-juiz-de-fora/1380937/ lei-6087-1981-juiz-de-fora-mg.html >. 8 Mar. 2013.

Juiz de Fora. Lei no 6.910 de 31 de maio de 1986. Dispõe sobre o ordenamento do uso e ocupação do solo no município de Juiz de Fora. <http://www.leismunicipais.com.br/legislacaode-juiz-de-fora/1060646/lei-6910-1986-juiz-de-fora-mg. html>. 8 Mar. 2013.

Juiz de Fora. Lei no 9.811 de 27 de junho de 2000. Institui o plano diretor de desenvolvimento urbano de Juiz de Fora. $<$ http://www.leismunicipais.com.br/legislacao-de-juiz-defora/1391368/lei-9811-2000-juiz-de-fora-mg.html >. 28 Jan. 2013.
Juiz de Fora. Lei no 11.817 de 04 de agosto de 2009. Altera a Lei Municipal no 6.087, de 04 de dezembro de 1981, e dá outras providências. <http://www.leismunicipais.com.br/ legislacao-de-juiz-de-fora/973791/lei-11817-2009-juiz-defora-mg.html>. 28 Jan. 2013.

Lial, S. W.; Gau, H. S.; Lai, W. L.; Chen, J. J.; Lee, C. G. Identification of pollution of Tapeng Lagoon from neighbouring rivers using multivariate statistical method. Journal of Environmental Management, v.88, p.286-292, 2008.

Machado, P. J. O. Crescimento urbano e ocupação de mananciais: $\mathrm{O}$ caso de Juiz de Fora/MG. In: Fórum Ambiental da Alta Paulista, 2, 2006, Estância Turística de Tupã. Resumos... Estância Turística de Tupã: ANAP/FACCAT/UNESP, 2006. CD Rom

Mendonça, E. C. C. N.; Souza, P. S. de. Aplicação da técnica de análise de componentes principais para caracterização de águas de poços artesianos de áreas urbanas de Goiânia e Aparecida de Goiânia. Plurais, v.1, p.19-36, 2011.

Padial, P. R.; Pompêo, M.; Moschini-Carlos, V. Heterogeneidade espacial e temporal da qualidade da água no reservatório Rio das Pedras (Complexo Billings, São Paulo). Ambi-Agua, v.4, p.35-53, 2009.

Ribeiro, C. R.; Pizzo, H. S. Avaliação da sustentabilidade hídrica de Juiz de Fora/MG. Mercator, v.10, p.171-88, 2011.

Silva, A. P. S.; Dias, H. C. T.; Bastos, R. K. X.; Silva, E. Qualidade da água do Reservatório da usina hidrelétrica (UHE) de Peti, Minas Gerais. Revista Árvore, v.33, p.1063-1069, 2009.

Sperling, M. von. Princípios do tratamento biológico de águas residuárias: Introdução à qualidade das águas e ao tratamento de esgotos, v.1, 3.ed. Departamento de Engenharia Sanitária e Ambiental. Universidade Federal de Minas Gerais. Belo Horizonte: Imprensa Universitária da UFMF, 2005. 452p.

Toledo, L. G. de; Nicolella, G. Índice de qualidade de água em microbacia sob uso agrícola e urbano. Scientia Agrícola, v.59, p.181-186, 2002.

Tundisi, J. G.; Tundisi, T. M. Impactos potenciais das alterações do Código Florestal nos recursos hídricos. Biota Neotropica, v.10, p.67-75, 2010.

Vanzela, L. S.; Hernandez, F. B. T.; Franco, R. A. M. Influência do uso e ocupação do solo nos recursos hídricos do Córrego Três Barra, Miranópolis. Revista Brasileira de Engenharia Agrícola e Ambiental, v.14, p.55-64, 2010. 